\title{
Sonderpädagogische Fördersysteme
}

\author{
Justin J.W. Powell und Lisa Pfahl
}

Sonderpädagogische Förderung soll benachteiligte, beeinträchtigte und behinderte Schüler und Schülerinnen in ihrem Lernen und in ihrer sozialen Entwicklung unterstützen. Schüler und Schülerinnen werden in den Bereichen Lernen, Sehen, Hören, Sprache, körperliche und motorische Entwicklung, geistige Entwicklung sowie emotionale und soziale Entwicklung sonderpädagogisch gefördert. Um ihre individuellen Lernziele zu erreichen und ihre größtmögliche Teilhabe am Leben in der Schule und in der Gesellschaft zu ermöglichen, erhalten diese Kinder und Jugendliche zusätzliche Aufmerksamkeit und Ressourcen. Diese Leistungen werden jedoch in sehr unterschiedlichen Schulformen angeboten - die allermeisten sonderpädagogischen Unterstützungsleistungen werden noch in Sonder- bzw. Förderschulen bereitgestellt. Parallel dazu gibt es seit den 1970er-Jahren, verstärkt seit den 1980er-Jahren, in manchen Bundesländern stetig - aber graduell - ausgebaute Programme gemeinsamen Unterrichts behinderter und nichtbehinderter Kinder (Eberwein 2009). Dennoch gibt es die inklusive Bildung - ein inzwischen als globale Norm gesetztes Ziel und in Deutschland durch Ratifizierung der Behindertenrechtskonvention 2009 eine gesetzgeberische Selbstverpflichtung - nur vereinzelt, nicht flächendeckend.

Deutschland war einst Pionier des Ansatzes der Hilfs- und Sonderschule, welcher vielen als beeinträchtigt wahrgenommenen Kindern überhaupt erst den Weg ins Bildungssystem ebnete. Heute stellt Deutschland im Ländervergleich ein außergewöhnliches Land dar - aufgrund der Persistenz des hochdifferenzierten segregativen Sonderschulwesens. Dabei wird die getrennte Beschulung von Kindern, sei es begründet durch eine medizinisch-klinische Diagnostik oder legitimiert durch eine professionelle pädagogische Autorität, zunehmend kontrovers diskutiert (Stöppler 2010; Wocken 2010). Eine Transformation hin zu einem inklusiven Bildungssystem, in dem alle Schüler und Schülerinnen möglichst lange gemeinsam lernen, hat es jedoch bisher nur regional bedingt gegeben. Beständig scheint vor allem die Kluft zwischen Integrations-Rhetorik und anhaltender gesellschaftlicher und schulischer Realität der Aussonderung und Separation.

Im deutschen Bildungswesen werden nach wie vor Schüler und Schülerinnen mit sonderpädagogischem Förderbedarf zum Großteil in Sondereinrichtungen oder -lerngruppen unterrichtet. Seit Jahrzehnten verlässt die große Mehrheit der Absolventen und Absolventinnen von Sonderschulen die Schule ohne Hauptschulabschluss - Tendenz steigend: im Jahr 2008 erhielten vier von fünf Sonderschülern und Sonderschülerinnen keinen Schulabschluss (Krappmann/Leschinsky/Powell 2003: 773; Werning/Reiser 
2008: 534; KMK 2010a). Folglich wird dieser Personengruppe somit auch der Zugang zum beruflichen Ausbildungssystem, dem Hochschulsystem und dem Arbeitsmarkt häufig versagt. Für den Zugang zum Arbeitsmarkt und damit für die Lebenschancen insgesamt sind Bildung und Ausbildung entscheidend, wie die Lebensverlaufsforschung belegt (Mayer 2009). Schulische Bildung, der sich eine berufliche Ausbildung anschließt, ist mehr denn je die Mindestvoraussetzung für eine existenzsichernde, stetige Beschäftigung geworden (Solga 2005). Damit rücken Fragen nach dem Zugang zu bestimmten Schulformen, den dort angeboten Lernopportunitäten und den Abschlussraten, die je nach Bundesland, Schulform und Schule erheblich variieren, bei der Betrachtung der sonderpädagogischen Fördersysteme ins Zentrum.

Daher wird in diesem Beitrag aus der Perspektive der Bildungssoziologie ein kritischer Blick auf die sonderpädagogischen Fördersysteme, die Disziplinbildung der Sonderpädagogik und die Folgen von Sonderbeschulung für behinderte und benachteiligte Schülergruppen entwickelt. Dazu werden einleitend einige Kennzeichen sonderpädagogischer Förderung zusammengefasst und in historischer Perspektive wichtige Entwicklungen sonderpädagogischer Fördersysteme nachgezeichnet. Im Mittelpunkt des Beitrags steht die Ausdifferenzierung der sonderpädagogischen Arbeitsfelder und die zahlenmäßige Verteilung klassifizierter Schüler und Schülerinnen auf Sonderschulen und allgemeine Schulen anhand der aktuellen amtlichen Statistik. Um den besonderen Entwicklungspfad Deutschlands zu verdeutlichen, werden außerdem Ergebnisse ländervergleichender Studien dargestellt sowie Erkenntnisse diskursanalytischer Forschung zur Sonderpädagogik präsentiert, die die Persistenz schulischer Segregation professionssoziologisch erklärt. Abschließend wird eine lebenslaufs- bzw. biografieanalytische Perspektive auf die Folgen schulischer Segregation eingenommen. Dabei werden die sozialen Stigmatisierungsprozesse sowie die Subjektivierungswirkung der sonderpädagogischen Klassifizierungspraktiken beschrieben. Die Folgen schulischer Segregation stehen den Zielen eines inklusiv ausgerichteten Bildungswesens entgegen, das auf einer Förderung von Kindern und Jugendlichen mit ihren individuellen Bedürfnissen durch einen produktiven Umgang mit der Vielfalt der Lernvoraussetzungen in gemeinsamen Schulen und im gemeinsamen Unterricht basiert. In einem Ausblick wird die seit 2009 in Deutschland gesetzlich bindende Behindertenrechtskonvention vorgestellt, die Inklusion von allen Kindern, Jugendlichen und Erwachsenen im Bildungswesen als Menschenrecht festschreibt.

\section{Von der Exklusion zur Inklusion? Die anhaltende Segregation und Separation bei gleichzeitiger Ausweitung der Integration}

Wie erfolgreich sind die unterschiedlichen Schul- und Unterrichtsformen - die von Unterricht im Krankenhaus über Sonderschulen hin zu mobilen Diensten oder Kooperationsklassen bis zum vollzeitigem gemeinsamen Unterricht reichen - in der Un- 
terstützung von Kindern und Jugendlichen mit sonderpädagogischem Förderbedarf? Diese Frage kann bisher mangels repräsentativer Längsschnittuntersuchungen zum direkten Vergleich aller Schulformen und der langfristigen Entwicklung der Lernenden für die Bundesländer Deutschlands nicht abschließend beantwortet werden. Analysen, die die Qualität und Ergebnisse verschiedener Ansätze und Fördersysteme vergleichen, um "good practices" festzustellen und zu verbreiten, fehlen in der bildungs- und erziehungswissenschaftlichen Forschung weitgehend. Doch auch wenn die zur Verfügung stehende quantitative Datenbasis dieses Beitrags - die amtliche Statistik - nach wie vor unbefriedigend bleibt (Cloerkes 2003), erlaubt sie es uns dennoch, die Strukturen und die Herausforderungen dieses Bildungsbereichs zu verdeutlichen. Die Forschung zu schulischer Integration und inklusiver Bildung zeigt zudem, dass erfolgreiche und realisierbare Alternativen zur Sonderbeschulung existieren und ausgeweitet werden (können) - auch in Deutschland.

Die sonderpädagogischen Fördersysteme Deutschlands können im Hinblick auf die Förderorte grundlegend unterschieden werden; dabei vollzieht sich in vielen westlichen Ländern ein gradueller Übergang von der Exklusion zur vollständigen Inklusion entlang eines Kontinuums von Segregation (Trennung zwischen Gebäuden), über Separation (Trennung innerhalb eines Gebäudes) und Integration (teilweise gemeinsamer Unterricht) hin zu vollständiger Inklusion (gemeinsamer Unterricht). Eine Überwindung der prinzipiellen Exklusion, wie sie in den 1960er-Jahren in Deutschland erreicht wurde, ist demnach nur der erste Schritt auf dem Weg zur größtmöglichen Teilhabe an formal organisierten Lernmöglichkeiten. Der gegenwärtige Ausbau des Sonderschulsystems bei gleichzeitiger Zunahme von schulischer Integration steht der aktuellen bildungspolitischen Zielsetzung der Inklusion aller Schüler und Schülerinnen in gemeinsamen Klassen entgegen; dabei verspricht die Überwindung der Segregation über die Separation und die Integration hin zur Inklusion eine kontinuierliche Verminderung der Stigmatisierung der Schüler und Schülerinnen (Powell 2011). Länder wie Norwegen oder Italien beweisen, dass es möglich ist, auf unterschiedliche Lernorte größtenteils zu verzichten - zugunsten einer inklusiven Schule für alle. Deutschland ist noch sehr weit entfernt vom Ziel der Inklusion. Dies hängt sowohl mit der Institutionalisierung der Fördersysteme im 20. Jahrhundert zusammen als auch mit den Professionsinteressen der Sonderpädagogik sowie der allgemeinen Pädagogik (Pfahl 2011).

Die bestehenden sonderpädagogischen Fördersysteme sind Teil des allgemeinen Bildungswesens, bildeten jedoch über die Zeit hinweg ein eigenständiges, hochdifferenziertes Gefüge an Einrichtungen aus. Sonderpädagogische Förderung erfährt weltweit eine starke Ausweitung. Trotz ihres Ursprungs im Zuge der Aufklärungsbewegung (noch vor Einführung der allgemeinen Schulpflicht) und ihrem prägenden Einfluss auf das Bildungssystem insgesamt werden sonderpädagogischen Fördersystemen sowohl in der Bildungsforschung als auch in der Bildungspolitik (noch) zu wenig Aufmerksamkeit geschenkt (Richardson/Powell 2011). Oft werden sie fachlich, räumlich und diskursiv marginalisiert oder gar als Teil klinischer Institutionen verstanden. 
Voraussetzung der Analyse der heute existierenden sonderpädagogischen Fördersysteme ist eine Begriffsbestimmung der unterschiedlichen Förderorte innerhalb des Bildungswesens. Dies ist umso wichtiger, als in Deutschland, wie in den meisten anderen Ländern Europas, eine Auflösung der traditionellen Dichotomie von Sonder- versus Regelschule $\mathrm{zu}$ beobachten ist und sonderpädagogische Förderung an unterschiedlichen Lernorten bereitgestellt wird (EADSNE 2003). Die Autoren dieses Beitrags schlagen in Anlehnung an den gegenwärtigen Gebrauch vor, die Beschulung von behinderten, beeinträchtigten und benachteiligten Kindern und Jugendlichen in einem räumlich getrennten Sonderschulwesen als Segregation, den (nahezu) vollständigen Unterricht in separaten Klassen und Lerngruppen an allgemeinen Schulen als Separation, den größtenteils gemeinsamen Unterricht als Integration und den auf Vielfalt und Differenzen jeglicher Art (etwa Schicht, Geschlecht, Migrationshintergrund, gemessene Intelligenz $u$. a.) ausgerichteten vollständig gemeinsamen Unterricht an allgemeinen Schulen als Inklusion zu bezeichnen. Dabei gibt es in der inklusiven Bildung unterschiedliche Schwerpunkte, etwa in den Unterrichtszielen, der Gruppenzusammensetzung innerhalb des Klassenzimmers oder der Individualisierung des Unterrichts auf Basis von Förderplänen (u. a. Index for Inclusion nach Booth/Ainscow 2000; Boban/Hinz 2003). Wichtig sind aber auch geteilte Prinzipien einer Pädagogik der Vielfalt wie die Akzep$\tan z$ von Verschiedenheit, der gleichberechtigten Teilhabe in den Lerngruppen als auch der demokratischen Mitbestimmung von Schülern und Schülerinnen, Eltern und Lehrkräften in ihrer Schule (vgl. Giroux 1997; Prengel 2006; Seitz 2003).

Die Expansion sonderpädagogischer Fördersysteme wurde durch die Ausweitung der allgemeinen Schulpflicht, der Ausrichtung des Bildungswesens an der Leitidee einer grundlegenden Bildung für alle Bürger und Bürgerinnen, aber auch durch die Etablierung der sonderpädagogischen Profession und ihre starke Interessenvertretung gesichert. Wurden benachteiligte und behinderte Kinder und Jugendlichen lange vom Schulbesuch komplett ausgeschlossen, wurde ihr Einschluss in das Bildungswesen durch die Gründung neuer Organisationsformen vorangetrieben, die die „Bildsamkeit“ unterschiedlicher Zielgruppen berücksichtigten: zunächst in einigen "Blinden“- und "Taubstummen“-Schulen und durch Einrichtung von „Idiotenschulen“ oder „Hilfsklassen" für arme, verwahrloste und kranke Kinder (Ellger-Rüttgardt 2008). Zu Beginn des 2o. Jahrhunderts wurde eine flächendeckende Versorgung mit Hilfsschulen in Deutsch land angestrebt - und schließlich erreicht. Ihre Aufgabe war es, die Volksschulen von den "schwach begabten“ Schülern und Schülerinnen zu „entlasten“ und diesen an den Hilfsschulen besonderen Schutz und (körperliche) Fürsorge zukommen zu lassen. Der Ausbau der Hilfsschulen als eigenständiges Schulwesen ging mit der Anerkennung der Hilfsschullehrkräfte als eigenständiger Berufsstand einher (Hänsel 2005). Die seit 1908 erscheinende Die Hilfsschule wurde zur Zeitschrift des Berufsverbandes der Sonderpädagogik und dokumentiert die Geschichte der Sonderpädagogik vor, während und nach dem Nationalsozialismus: „Erfolg, Niedergang, Neuanfang" (Möckel 1998). Nach der Etablierung der eigenständigen Schulform zu Beginn des 20. Jahrhunderts wurde das 
Hilfsschulwesen in der Zeit des Dritten Reichs auch zur Erfassung von kranken, behinderten und benachteiligten Personen genutzt. Kinder und Jugendliche aus pädagogischen Einrichtungen wurden teilweise in Kollaboration mit den Nationalsozialisten den grausamen und tödlichen Maßnahmen der "Rassenhygiene" ausgeliefert, teilweise wurden sie von Pädagogen und Pädagoginnen vor nationalsozialistischen Übergriffen versteckt und geschützt (Ellger-Rüttgardt 2008).

Die Restauration des sonderpädagogischen Fördersystems nach 1945 und der Rückgriff auf Modelle der Weimarer Republik ebneten den Weg zum stetigen Ausbau über das 20. Jahrhundert hinweg. Insbesondere ab den 1960er Jahren begann in Deutschland durch die Einrichtung zahlreicher neuer Schulformen und einer Ausweitung des Angebots in den vor- und nachschulischen Bereich eine weltweit nahezu einzigartige Ausdifferenzierung der sonderpädagogischen Fördersysteme (Powell 2011). Die etwas später einsetzende und erstarkende Bestrebung von Eltern förderbedürftiger Kinder, diese an allgemeinen Schulen zu integrieren, führte zur Einrichtung von Modellschulen des gemeinsamen Unterrichts, aber bislang nicht zu einer Abkehr des Ansatzes der separaten Beschulung (Preuss-Lausitz 2001). Die Professionalisierungsgeschichte der deutschen Sonderpädagogik blieb über das 20. Jahrhundert hinweg eng an den Ausbau von Sonderschulen für arme, auffällige, kranke und behinderte Kinder und Jugendliche geknüpft (Moser et al. 2008). Die Separation der Kinder und Jugendlichen und ihre schulische Segregation sicherte den Berufsangehörigen der Sonderpädagogik die Alleinzuständigkeit für die Schülergruppen an den für sie geschaffenen Sonderschuleinrichtungen, wie eine Diskurs- und Machtanalyse der Sonderpädagogik für den Förderschwerpunkt Lernen zeigt (Pfahl 2011). Bis heute besitzt die Sonderpädagogik ein Interesse an der Erhaltung separierender Einrichtungen aus Sorge um den Verlust der Autonomie und ihrer teilweise ökonomischen Besserstellung gegenüber Lehrkräften von Grund-, Haupt- und Realschulen und stützt damit die Segregation.

\section{Das deutsche Fördersystem im internationalen Kontext}

Im Vergleich mit anderen europäischen Staaten liegt Deutschland mit dem Anteil von Schülern und Schülerinnen, denen sonderpädagogischer Förderbedarf attestiert wird, im Mittelfeld. Der Anteil der Schüler und Schülerinnen mit sonderpädagogischem Förderbedarf, die an Sonderschulen unterrichtet wird, ist hingegen im europäischen Vergleich sehr hoch. Dabei variieren sowohl die Förderquoten als auch die Segregationsraten von Schülern und Schülerinnen mit sonderpädagogischem Förderbedarf regional. Im Hinblick auf die europäischen Nachbarländer und die USA wird klar, dass die Bandbreite der Gruppengrößen sehr unterschiedlich ist - von einem Prozent in Italien bis zu einem Fünftel in Finnland (siehe Abbildung 1: auf der linken Seite befinden sich die Gesamtförderquoten in Prozent, diese geben Aufschluss über den Anteil aller Schüler und Schülerinnen, die als förderbedürftig klassifiziert sind). Noch größere 
Varianzen zeigen sich aufgrund der grundlegend verschiedenen Bildungssysteme, von denen einige nahezu vollständig segregieren (wie z. B. Belgien) bis hin zu Italien, wo nur vereinzelt an Sonderschulen unterrichtet wird (siehe Abbildung 1: auf der rechten Seite befinden sich die Segregationsraten; diese geben Aufschluss über den Anteil der Schüler und Schülerinnen mit sonderpädagogischem Förderbedarf, die schulisch segregiert, d.h. an Sonderschulen unterrichtet werden).

\section{Abbildung 1 Förderquoten und Segregationsraten in Deutschland, Europa und USA}

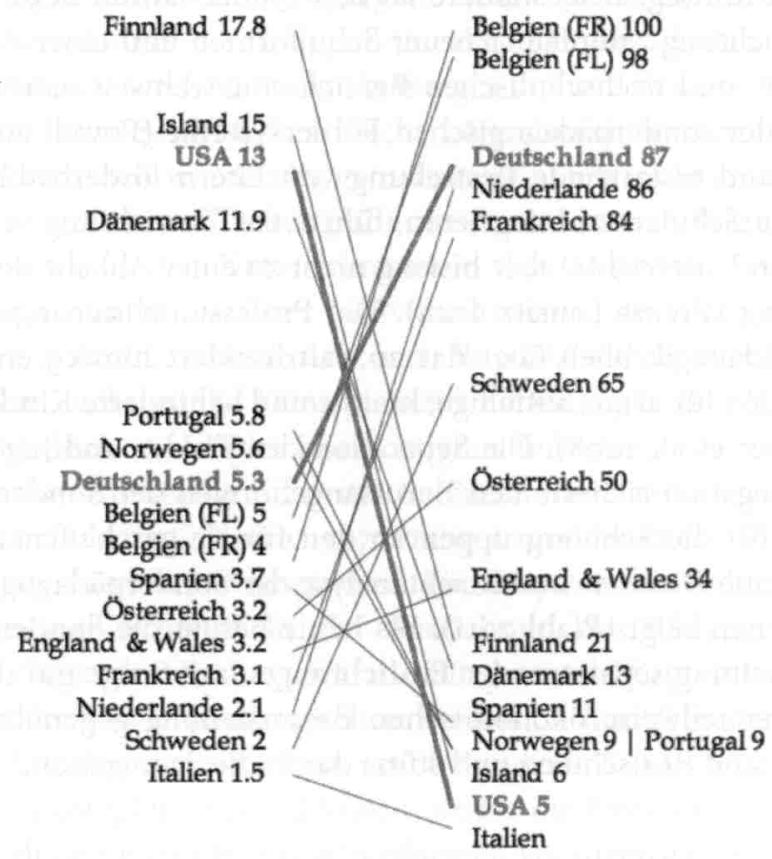

Quelle: Powell/Pfahl 2009 auf Basis von EADSNE (2003), Eurydice (2002), US DoED (2005).

An dem Vergleich von Deutschland und den USA wird exemplarisch deutlich, dass auch zwei Länder, deren sonderpädagogische Fördersysteme ursprünglich relativ ähnlich waren, zunehmend divergieren, sowohl in den Partizipations- und Segregationsraten als auch in den Absolventenzahlen (Powell 2011). In den USA gibt es heute eine relativ große klassifizierte Gruppe, die größtenteils in Regelschulen integriert ist (jedoch nicht ganztägig in Regelklassen inkludiert ist). In Deutschland wird eine viel kleinere Gruppe klassifiziert, diese wird dann jedoch fast ausschließlich räumlich getrennt unterrichtet. Sonderpädagogische Fördersysteme nehmen innerhalb Europas und den USA sehr verschiedene Formen an. Ein schulrechtlich anerkannter Förderbedarf von Kindern und Jugendlichen mit Benachteiligungen und Behinderungen kann eine Ausnahme sein - oder zur Selbstverständlichkeit an Regelschulen werden. 
Grundlegend sind drei Modelle sonderpädagogischer Fördersysteme zu unterscheiden: Das „binäre Modell“, das „Kontinuum-Modell“ und das „Inklusions-Modell“. Diese differenzieren sich nach den Lernorten und den Gruppengrößen; sie weisen Segregationsraten von nahezu o bis $100 \%$ auf (siehe Abbildung 1). Der internationale Vergleich kontrastiert das „binäre Modell“ im deutschen Fall (mit der strikten Trennung zwischen allgemeinen Schulen und Sonderschulen) und das "Kontinuum-Modell“ im amerikanischen Fall (in dem vielfältige Organisationsformen existieren). Keines der beiden Länder hat eine unitäre, auf die „Inklusion aller Kinder" ausgerichtete Schulstruktur. Ein solches „Inklusions-Modell, “ bei dem alle Schüler und Schülerinnen den größten Teil des Schulalltags gemeinsam unterrichtet werden, findet sich z. B. in Italien, Island und Norwegen, wobei sich die Ausgestaltung der Unterstützungssysteme und ihre Leistungen unterscheiden. Der deutsch-amerikanische Vergleich zeigt zudem, dass es nicht ausreicht, nur über das Entweder/Oder der Segregation und Inklusion zu sprechen, da es in den USA und zunehmend auch in Deutschland eine Reihe unterschiedlicher Organisationsformen der Förderung gibt.

\section{Klassifizierung und Stigmatisierung: das Etikettierungs-Ressourcen-Dilemma}

Im Zuge der Einschulungsuntersuchungen, des Grundschulunterrichts oder der Zuweisung in die verschiedenen Schultypen der Sekundarstufe wird im segregativen deutschen Bildungswesen „auffälligen" oder beeinträchtigten Kindern und Jugendlichen ein sonderpädagogischer Förderungsbedarf attestiert, dem in der Regel eine Sonderschulüberweisung folgt. Die sonderpädagogische Förderung wurde $1994 \mathrm{zu}$ sonderpädagogischen Förderschwerpunkten umstrukturiert, aber die organisatorische Differenzierung und Stratifizierung der Schulformen ist geblieben. Der Prozess des sonderpädagogischen Klassifizierens wird in erster Linie durch die pädagogische Praxis in Schulen bestimmt, wobei international anerkannte Klassifikationssysteme der Weltgesundheitsorganisation wie der "International Classification of Functioning, Disability and Health" und national bestimmte Klassifikationssysteme einflussreich wirken (Hirschberg 2009).

Sonderpädagogische Fördersysteme beruhen auf dem Versuch, den Förderbedarf von Kindern und Jugendlichen zu typisieren und zu klassifizieren. In einem stratifizierten Bildungswesen wie dem Deutschlands werden Schülerinnen und Schüler von Beginn ihrer Schulzeit an biologischen, kognitiven oder sozialen Standards gemessen, um festzustellen, ob sie von den durchschnittlich zu erwartenden Fähigkeiten, Leistungen oder Verhaltensweisen abweichen. In den Bildungseinrichtungen sind es im Besonderen die Prozesse des Klassifizierens von Schülern und Schülerinnen, die dazu führen, dass Einzelne als „defizitär“ oder „abweichend“ wahrgenommen werden. Zugleich stellt das Klassifizieren von Schülern und Schülerinnen den Mechanismus dar, an den die Vergabe von Ressourcen und die Gewährung von Rechten in Bildungseinrichtungen geknüpft ist. Der Prozess der Klassifizierung von Schülergruppen stellt somit eine Schlüs- 
selrolle für die Schullaufbahn von Kindern und Jugendlichen dar. Auf der Schulebene wird dies als „Etikettierungs-Ressourcen-Dilemma“ beschrieben (Füssel/Kretschmann 1993), da die Bereitstellung zusätzlicher Ressourcen bislang durch eine Klassifikation der Schüler und Schülerinnen legitimiert werden muss, die ihre Diskreditierbarkeit erhöht. Im deutschen sonderpädagogischen Fördersystem stellt in der Regel die Sonderschule den Ort dar, an dem zusätzliche Ressourcen zur Verfügung gestellt werden. Mit der Klassifizierung eines sonderpädagogischen Förderbedarfs geht deshalb regelmäßig eine Stigmatisierung und Segregierung der Bedürftigen einher.

Die Feststellungsverfahren des sonderpädagogischen Förderbedarfs werden von Lehrkräften, Ärzten und Psychologen vorgenommen. Diese zeitaufwändigen Verfahren setzen in der Regel erst nach größeren Leistungsrückständen der Schüler und Schülerinnen ein und gehen größtenteils erst nach erfolgter Sonderschulüberweisung mit einer zusätzlichen Ressourcenzuwendung einher - darin besteht eine „doppelte Diskriminierung" dieser Kinder und Jugendlichen. Die Verfahren sind nicht "ergebnisoffen" und lassen die Möglichkeit einer besonderen Förderung in der allgemeinen Schule, wie beispielsweise dem Erstellen eines individuellen Lehrplans, meist ungenutzt (Kottmann 2006). Die von den Feststellungsverfahren erfassten Schüler und Schülerinnen stellten schon immer eine sozial sehr selektive Gruppe dar (Hofsäss 1993). Ältere Studien belegen, dass die Mehrheit aller Sonderschüler und -schülerinnen mit sonderpädagogischem Förderbedarf, sozial und ökonomisch benachteiligt ist (z. B. Hofmann 1930; Begemann 1970; Wocken 2000). Sehr viele Schüler und Schülerinnen der Sonderschule für Lernbehinderte wachsen mit erwerbslosen oder gering qualifizierten Eltern auf (Pfahl 2004). Kinder und Jugendliche mit Migrationshintergrund sind dabei deutlich überrepräsentiert (Kornmann 2003). Ähnlich wie in Deutschland sind ethnische Minderheiten in den USA, Kanada oder Neuseeland seit Jahrzehnten in sonderpädagogischen Maßnahmen überrepräsentiert (Gabel et al. 2009). Zudem tragen Jungen ein größeres Risiko, an einer Sonderschule segregiert zu werden - vor allem an Sonderschulen für Lernbehinderte, an der sie zwei Drittel der Schülerschaft darstellen (Faulstich-Wieland 2004). Diese Indikatoren verdeutlichen die Bildungsungleichheiten an der Schnittstelle zwischen Sonder- und Regelschule.

\section{Aktuelle Entwicklungen sonderpädagogischer Förderung in Deutschland}

2008 gab es 482415 Schüler und Schülerinnen, die in Sonder- oder Förderschulen und allgemeinen Schulen in Deutschland sonderpädagogische Förderung erhalten haben (KMK 2010a). Damit wurde die höchste Gesamtförderquote jemals erreicht: über $6 \%$ aller Schüler und Schülerinnen erhielten an Sonder- oder Förderschulen sowie an allgemeinen Schulen eine sonderpädagogische Förderung. Es wurden 88924 Schülerinnen und Schüler mit sonderpädagogischem Förderbedarf gezählt, die allgemeine Schulen besuchten. Der Anteil dieser sogenannten Integrationsschüler/innen (erst seit der Jahr- 
tausendwende in der amtlichen Bundesstatistik ausgewiesen) ist seit 2000 von 12,4\% auf $18,4 \%$ im Jahr 2008 graduell angestiegen, wobei diese Zahlen große Unterschiede in der Art und Dauer der zugeteilten Förderung verdecken. Die Mehrheit lernte in Grundschulen (59,5\%), in Hauptschulen (17,0\%) oder in Integrierten Gesamtschulen (6,0\%). Ebenfalls waren diese in allgemeinen Schulen geförderten Kinder recht konzentriert auf wenige Kategorien, nämlich die Förderschwerpunkte Lernen $(44,8 \%)$, emotionale und soziale Entwicklung (22,4\%) und Sprache (15,6\%) (KMK 2010a).

Die Verteilung aller sonderpädagogisch geförderten Kinder und Jugendlichen unter die seit 1994 gültigen Schwerpunkte hat sich graduell verändert, wobei insbesondere der größte Schwerpunkt (Lernen) rückläufig ist und der drittgrößte emotionale und soziale Entwicklung ansteigt (aktueller Anteil und absolute Zahlen für 2008 in Klammern): Üblicherweise wird unterschieden zwischen dem Förderschwerpunkt Lernen (43,7\%; 210 952) und sonstigen Förderschwerpunkten $(56,3 \% ; 271463)$. Zur Oberkategorie "Sonstige" gehören: geistige Entwicklung (16,0\%); emotionale und soziale Entwicklung $(11,5 \%)$; Sprache (10,6\%); körperliche und motorische Entwicklung (6,5\%); Förderschwerpunkt übergreifend bzw. ohne Zuordnung (5,0 \%); Hören (3,1\%); Kranke (2,1\%); Sehen (1,5\%). Diese Schüler und Schülerinnen wurden von insgesamt 65014 Lehrern und Lehrerinnen in 40131 Klassen aller Förderschwerpunkte unterrichtet. Ungefähr zehn Schüler und Schülerinnen werden pro Klasse unterrichtet, und dieses Schüler/ Lehrer-Verhältnis von $6 \mathrm{zu} 1$ ist das bisher niedrigste (KMK 2010a).

Trotz Entwicklungen hin zur verstärkten schulischen Integration wird bis heute der überwiegende Teil der sonderpädagogisch geförderten Schülerinnen und Schüler in Sonderschulen unterrichtet. Auch die große Chance der deutschen Wiedervereinigung wurde nicht genutzt, um die schulische Integration flächendeckend auszuweiten. Im Gegenteil sind es insbesondere die neuen Bundesländer, die hohe Sonderbeschulungsraten aufweisen (KMK 2010a).

Dennoch zeigen die großen Disparitäten in den Sonderschulbesuchsquoten von 1992 bis 2008 die Dynamiken des Besuchs dieser Schulform. Insgesamt ist, getrieben von 13 Bundesländern, die Bundesquote von $4,18 \%$ auf $4,92 \%$ gestiegen, wobei die Erhöhung besonders markant war in Thüringen $(7,47 \%)$, Sachsen-Anhalt $(8,73 \%)$ und Mecklenburg-Vorpommern (9,15\%), dort also zwei bis drei Mal so viele Schüler und Schülerinnen eine Sonderschule besuchen wie in Schleswig-Holstein $(3,12 \%)$, wo ein signifikanter Rückgang erreicht wurde (siehe Tabelle 1). Auch die Stadtstaaten Hamburg und Bremen konnten leichte Rückgänge der Sonderschulbesuchszahlen verzeichnen. Diese Fälle belegen, dass ein Wandel in beide Richtungen möglich ist. Sowohl über die Zeit als auch räumlich zeigen diese Differenzen, dass - so wichtig individuelle Merkmale der Lernenden auch sind - die kulturellen und sozioökonomischen Rahmenbedingungen, die politischen Entscheidungen und die Ermessensspielräume in Schulen bei der Überweisungspraxis von enormer Bedeutung sind. Durch die Einnahme einer räumlich und historisch vergleichenden Perspektive können darüber hinaus sowohl die schulischen Strukturen als hochgradig variabel als auch die existierenden Behinderungska- 
Tabelle 1 Sonderschulbesuchsquoten, Bundesländer, 1992-2008

\begin{tabular}{lccccccccc}
\hline Bundesland & 1992 & 1994 & 1996 & 1998 & 2000 & 2002 & 2004 & 2006 & 2008 \\
\hline BW & 4.47 & 4.39 & 4.31 & 4.27 & 4.31 & 4.48 & 4.51 & 4.55 & 4.71 \\
BY & 3.99 & 4.23 & 4.45 & 4.65 & 4.65 & 4.64 & 4.49 & 4.42 & 4.62 \\
BE & 3.75 & 3.55 & 3.56 & 3.80 & 4.06 & 4.35 & 4.55 & 4.47 & 4.36 \\
BB & 4.72 & 4.67 & 4.68 & 4.94 & 5.34 & 5.66 & 6.08 & 5.98 & 5.42 \\
HB & 4.69 & 4.43 & 4.15 & 4.11 & 4.13 & 3.73 & 3.87 & 4.22 & 4.61 \\
HH & 5.19 & 5.20 & 5.09 & 5.00 & 4.88 & 4.90 & 4.87 & 4.82 & 4.88 \\
HE & 3.14 & 3.17 & 3.26 & 3.43 & 3.68 & 4.00 & 4.17 & 4.28 & 4.31 \\
MV & 4.63 & 4.86 & 5.30 & 5.94 & 6.70 & 7.40 & 8.24 & 8.65 & 9.15 \\
NI & 3.69 & 3.71 & 3.75 & 3.91 & 4.09 & 4.31 & 4.36 & 4.29 & 4.40 \\
NW & 4.50 & 4.54 & 4.40 & 4.29 & 4.57 & 4.88 & 5.00 & 5.07 & 5.24 \\
RP & 3.27 & 3.26 & 3.31 & 3.41 & 3.64 & 3.84 & 3.88 & 3.84 & 3.77 \\
SL & 2.85 & 2.83 & 2.95 & 3.12 & 3.30 & 3.62 & 3.84 & 4.01 & 4.23 \\
SN & 4.01 & 4.38 & 4.77 & 5.13 & 5.45 & 5.92 & 6.50 & 6.91 & 6.90 \\
ST & 5.02 & 5.43 & 5.76 & 6.28 & 7.01 & 7.67 & 8.12 & 8.46 & 8.73 \\
SH & 4.71 & 4.59 & 4.24 & 4.07 & 4.06 & 3.93 & 3.75 & 3.54 & 3.12 \\
TH & 4.55 & 4.97 & 5.59 & 6.25 & 6.98 & 7.76 & 7.88 & 8.00 & 7.47 \\
\hline Deutschland & 4.18 & 4.26 & 4.32 & 4.43 & 4.61 & 4.80 & 4.85 & 4.85 & 4.92 \\
\hline
\end{tabular}

Quelle: KMK 2010a. NB: Niedrigste und höchste Anteile.

tegorien als soziale Konstrukte aufgezeigt werden: Demnach ist die Klassifikation von Schülern und Schülerinnen nicht nur mit klinischen oder individuellen Faktoren zu erklären, sondern sie erlaubt es, Rückschlüsse auf erstaunlich disparate Rahmenbedingungen der über das 2o. Jahrhundert massiv expandierten sonderpädagogischen Fördersysteme zu ziehen (Powell 2011).

\section{Folgen der Sonderbeschulung: Stigmatisierung und Fehlende Schulabschlüsse}

Die Klassifikation „förderbedürftig“ und die Überweisung an eine Sonderschule hat weitreichende Folgen für die Betroffenen. Schüler und Schülerinnen, die eine Sonderschule besucht haben, stehen mit dem Ende der Schulzeit vor großen Hürden, da sie in der Regel kein qualifizierendes Schulzertifikat besitzen und ihnen der Übergang in postsekundäre Bildung ohne solche Zertifikate weitestgehend verwehrt wird. Auch der Übergang in berufliche Bildung wird strukturell stark erschwert, denn nur einer bzw. 
Tabelle 2 Sonderschulabgänger/innen ohne Hauptschulabschluss, Bundesländer, 1992-2008 (\%)

\begin{tabular}{lccccccccc}
\hline Bundesland & 1992 & 1994 & 1996 & 1998 & 2000 & 2002 & 2004 & 2006 & 2008 \\
\hline BW & 74 & 76 & 75 & 77 & 80 & 80 & 78 & 78 & 78 \\
BY & 85 & 84 & 85 & 86 & 87 & 88 & 85 & 84 & 84 \\
BE & 84 & 84 & 81 & 80 & 66 & 60 & 65 & 58 & 61 \\
BB & 77 & 79 & 81 & 85 & 87 & 89 & 92 & 94 & 95 \\
HB & 85 & 85 & 79 & 85 & 89 & 84 & 84 & 79 & 81 \\
HH & 74 & 71 & 81 & 80 & 81 & 84 & 83 & 86 & 80 \\
HE & 89 & 88 & 90 & 89 & 87 & 86 & 87 & 83 & 87 \\
MV & 93 & 95 & 91 & 86 & 87 & 88 & 85 & 81 & 81 \\
NI & 81 & 79 & 80 & 78 & 78 & 80 & 79 & 75 & 76 \\
NW & 52 & 54 & 51 & 56 & 60 & 65 & 68 & 69 & 68 \\
RP & 85 & 83 & 82 & 80 & 82 & 83 & 78 & 78 & 76 \\
SL & 89 & 82 & 88 & 90 & 92 & 86 & 79 & 74 & 66 \\
SN & 6 & 93 & 91 & 86 & 88 & 85 & 85 & 82 & 82 \\
ST & 94 & 90 & 85 & 82 & 81 & 85 & 82 & 76 & 77 \\
SH & 95 & 96 & 97 & 96 & 97 & 98 & 98 & 97 & 97 \\
TH & 91 & 94 & 92 & 89 & 84 & 80 & 72 & 65 & 57 \\
\hline Deutschland & 73 & 78 & 78 & 78 & 79 & 80 & 79 & 77 & 76 \\
\hline
\end{tabular}

Quelle: KMK 2010a.

eine von fünf Abgängern bzw. Abgängerinnen verlässt die Sonderschule mit einem Abschluss - allerdings mit einer großen Varianz je nach Förderschwerpunkt. Davon erhalten die meisten Sonderschüler und -schülerinnen einen Hauptschulabschluss (2008: 21,5\%; 9978), einige wenige erreichen einen Realschulabschluss (2,1\%; 971), oder die (Fach-)Hochschulreife (o,1\%; 76) (KMK 2010a: xvi). Der Anteil der Abgänger und Abgängerinnen ohne Abschluss variiert innerhalb Deutschlands stark; im Jahr 2008 lag die Quote zwischen $57 \%$ in Thüringen oder $61 \%$ in Berlin über einen Mittelwert für Deutschland insgesamt von $76 \%$ bis hin zu $95 \%$ in Brandenburg oder $97 \%$ in SchleswigHolstein (vgl. Tabelle 2). Die Spannbreite reflektiert auch Unterschiede in den schulgesetzlichen Regelungen, Differenzen in der sozioökonomischen Zusammensetzung der Schülerschaft und Disparitäten in den Integrationsraten.

Im weiteren Lebensverlauf bleibt die Erwerbs- und Lebenssituation ehemaliger Sonderschüler und -schülerinnen zumeist prekär und durch Maßnahmen beruflicher (Wieder-)Eingliederung geprägt. Zusätzliche segregierende Maßnahmen bringen Menschen mit Behinderungen nicht langfristig in qualifizierte Arbeitsverhältnisse. Die berufli- 
chen Benachteiligungen der Sonderschulabgängerinnen und Schulabgänger vermag jedoch auch ein Zuwachs an Angeboten im sogenannten Übergangssystem nicht zu kompensieren, weil die Maßnahmen beruflich nicht qualifizieren (Baethge et al. 2007), und nur in Einzelfällen zur Berufsausbildung führen (Pfahl 2004). Die damit verbundenen Arbeitslosigkeits- und Armutsrisiken werden durch die laufenden sozialpolitischen Maßnahmen nur unzureichend kompensiert. Hingegen versprechen bildungspolitische Reformen, die gegen die Herstellung von „Bildungsarmut" (Allmendinger 1999) und auf schulische Inklusion gerichtet sind, nicht nur die Arbeitsmarktsituation von Menschen mit Behinderungen zu verbessern, sondern auch die Möglichkeit der sozialen Teilhabe. Ein Übergang in eine berufliche Ausbildung gelingt Schülern und Schülerinnen im Anschluss an die sonderpädagogische Förderung an Sonderschulen nur äußerst selten. Studien zum Schul- und Lebensverlauf von Abgängern und Abgängerinnen von Lernbehindertenschulen zeigen, dass nur sehr wenige von ihnen in eine Berufsausbildung gelangen (Pfahl 2004; Wagner 2005), wobei denjenigen jungen Erwachsenen, die integrativ beschult wurden, der Übergang tendenziell besser gelingt (Ginnold 2008). Es ist bekannt, dass sich selbst für Jugendliche, die auf der Sonderschule für Lernbehinderte einen Hauptschulabschluss erlangen konnten, der Sonderschulbesuch bei der Suche nach einem Ausbildungsplatz negativ auswirkt. Handlungs- und wissenssoziologische Forschungsansätze, die das Verhalten der Schüler und Schülerinnen einbeziehen, können erklären, weshalb dies so ist.

Viele Jugendliche antizipieren ihre Chancenlosigkeit und reagieren in dieser Situation mutlos - sie ziehen sich aufgrund ihrer geringen Chancen von den Anforderungen der Erwerbsgesellschaft zurück. Denn die Benachteiligungen von Sonderschülern und -schülerinnen liegen in den Bereichen familialer Herkunft, misslungener Schulkarriere und einer sozialen Stigmatisierung. Die Jugendlichen erfahren durch gesellschaftliche Zuschreibungen eine Abwertung (Marquardt 1975). Die negativen Fremdzuschreibungen beeinflussen die Selbstwahrnehmung der Jugendlichen und ihre Persönlichkeitsbildung; sie führen - vermittelt durch eine Beschränkung der personalen und sozialen Rollen, die die Betroffenen in sozialen Interaktionen einnehmen können - zur Schädigung ihrer sozialen Identität (Goffman 1975; Krappmann [1971] 2005). Mögliche und wahrscheinliche Auswirkungen der Stigmatisierung stellen extreme Anpassung oder gar Rebellion gegenüber aus ihrer Sicht unzumutbaren gesellschaftlichen Erwartungen dar. Diese Formen des Umgangs mit der Stigmatisierung versprechen in Bezug auf die berufliche Orientierung der Jugendlichen wenig Erfolg. Ein Stigma-Management, das ein passives "Vermittelt-werden“ in das berufliche Bildungssystem ermöglicht, führt ebenso wenig zu einer beruflichen Integration wie die Abwehr einer beruflichen Orientierung durch aktive Verhaltensweisen.

Dabei zeigen genealogisch-biografische Studien, dass als behindert klassifizierte Personen eine lebensweltliche und berufliche Orientierung ausbilden, die durch die existierenden diskursiven Behinderungszuschreibungen, mit denen sie sich identifizieren, 
geprägt sind (Freitag 2005). Ehemalige Sonderschüler und -schülerinnen verfolgen aufgrund zahlreicher negativer sozialer Erfahrungen Stigmatisierungsvermeidungsstrategien, d.h. sie gehen bestimmten sozialen Situationen wie Bewerbungen oder sozialen Vergleichen mit Gleichaltrigen aus dem Weg, um nicht diskreditiert zu werden. Sie greifen die ihnen durch die Institutionen angebotenen Beschreibungen der Hilfsbedürftigkeit als Selbsttechniken auf und richten teilweise ihr Handeln danach aus. Dies führt dazu, dass sie ihre bereits eingeschränkten Handlungsmöglichkeiten nicht wahrnehmen (dazu Pfahl 2011). Die Praxis der schulischen Segregation, die trotz fehlender formaler Bildungserfolge durch sonderpädagogische Förderung an Sonderschulen dadurch gerechtfertigt wird, dass Sonderschulen ein "geschütztes" und „schützendes" Territorium außerhalb der Leistungsansprüche des allgemeinen Bildungswesens darstellen, sozialisiert die Schüler und Schülerinnen als Adressaten der wissenschaftlichen Behinderungsdiskurse in reduzierte Erwartungen (Pfahl/Powell 2011). Die im Diskurs um Behinderung bereitgestellten Kategorien (z. B. „lernbehindert") dienen dabei der Selbsteinschätzung der Betroffenen. So fließt das an der Schule erworbene, subjektiv-biografisch angeeignete Wissen über Behinderung als "Störung“ oder „individuelles Defizit", in das berufliche und lebensweltliche Handeln der jungen Erwachsenen ein. Ehemalige Sonderschüler und -schülerinnen fügen sich in die ihnen angebotenen sozialen Positionen als „Hilfsbedürftige“ ein und verbleiben langfristig in abhängigen Subjektpositionen. Zugleich wird das segregative Fördersystem durch die pädagogischen und sonderpädagogischen Disziplinen und die von ihnen hervorgebrachten Diskurse um Behinderung und Begabung aufrechterhalten (Pfahl 2011) .

Der Großteil der ehemals sonderpädagogisch geförderten Jugendlichen und jungen Erwachsenen wird nach der Schule in separierende Maßnahmen der Berufsvorbereitung gelenkt (Baethge et al. 2007). In diesen beruflich nicht qualifizierenden Maßnahmen verbleiben die jungen Erwachsenen in der Regel über mehrere Jahre, wobei Einzelnen ein nachholender Eintritt in eine Berufsausbildung gelingt, die große Mehrheit jedoch ohne berufliche Qualifikation bleibt. Das deutsche sonderpädagogische Fördersystem trägt somit dazu bei, dass junge Erwachsene ohne Ausbildung bleiben. Ohne Schulabschluss bleiben junge Erwachsene mit ehemaligem sonderpädagogischen Förderbedarf in der Regel dauerhaft geringverdienend oder erwerbslos. Zwar bleiben auch in den anderen europäischen Ländern Menschen mit Behinderungen im späteren Lebensverlauf überdurchschnittlich häufig arbeitslos und abhängig von staatlichen Leistungen und staatlicher Fürsorge (Maschke 2008). Junge Erwachsene jedoch, die in einem inklusiv ausgerichteten Bildungswesen gemeinsam unterrichtet werden, erhalten auf diese Weise mehr Bildung und von Beginn an erhöhte Teilhabechancen, wie der internationale Vergleich gezeigt hat. Schulabgänger und -abgängerinnen aus inklusiv ausgerichteten Bildungssystemen erhalten häufiger qualifizierende Schulabschlüsse, es eröffnen sich ihnen bessere Arbeitsmarktchancen, und die Aussicht auf berufliche Partizipation ist weniger prekär (Knauer/Ramseger 2009). Auf die persistente 
Ausgrenzung von behinderten Personen in segregativen Bildungssystemen reagiert eine umfassende Menschenrechtskonvention, deren weltweite Ratifizierung seit 2006 erfolgt. ${ }^{1}$

\section{Einfluss der UN Behindertenrechtskonvention und Schritte hin zur inklusiven Bildung}

Die UN-Konvention über die Rechte behinderter Menschen (BRK), die 2009 von der Bundesregierung ratifiziert wurde, legt fest, die Diskriminierung von Menschen mit Behinderungen in allen Lebensbereichen $z u$ verbieten und ihnen die politischen, wirtschaftlichen, sozialen und kulturellen Menschenrechte zu garantieren sowie Teilhabe am gesellschaftlichen Leben zu ermöglichen. Die Konvention untermauert den Paradigmenwechsel vom medizinischen zum menschenrechtlichen Modell von Behinderung und ,stellt den vorläufigen Abschluss der schrittweisen Anerkennung des Rechts auf inklusive Bildung für Menschen mit Behinderung dar. Sonderschulen werden durch Art. 24 BRK zwar nicht kategorisch verboten, die systematische Aussonderung behinderter Personen aus dem allgemeinen Bildungssystem stellt allerdings eine Vertragsverletzung dar" (Degener 2009: 216-217). Nach Artikel 24 sollen alle Bereiche von Bildungssystemen inklusiv umgestaltet werden. Inklusiv heißt: Alle Lernenden werden gemeinsam unterrichtet. Die UN-Konvention geht damit über das seit 1994 im Grundgesetz verankerte Gebot „Niemand darf wegen seiner Behinderung benachteiligt werden“ (Artikel 3, Absatz 3 GG) hinaus, denn es legt unmissverständlich nahe, Menschen mit Behinderungen den Zugang zu allgemeinen und weiterführenden Regelschulen und zu tertiärer Bildung nicht länger zu verwehren, d.h. ausreichend für inklusive Bildung auf allen Ebenen zu sorgen. Vor Ratifizierung der BRK hatte das Bundesverfassungsgericht diese Prinzipien der individuellen Förderung und des Wahlrechts nicht nur gestärkt. Am 8. Oktober 1997 hat das oberste Gericht (1 BvR 9/97) entschieden, dass dieses Grundrecht nur dann einen Anspruch auf integrative Beschulung gewähre, wenn in einem Bundesland entsprechende Ressourcen zur Verfügung stehen, aber ein Bundesland könne nicht gezwungen werden, diese zur Verfügung zu stellen; Haushaltsvorbehalte gibt es noch in vielen Bundesländern (Füssel 2009). Zukünftig ist aber das Gebot der Gleichbehandlung im Sinne der BRK auszulegen, weil Bund, Länder, deutsche Gerichte und Schulbehörden diese neuen Maßstäbe zu berücksichtigen haben und die Grundrechte in Einklang mit der internationalen Konvention bringen müssen.

Auch wenn die Konvention ein völkerrechtlicher Vertrag ist, der durch ein Vertragsgesetz gemäß Artikel 59 II GG in deutsches Bundesrecht umgesetzt wird, liegen bisher nur wenige rechtskräftige Urteile in Bezug auf die BRK vor; diese werden durch die Monitoringstelle im Deutschen Institut für Menschenrechte dokumentiert und kom-

1 Seit 2006 haben über 100 Länder die Konvention ratifiziert, siehe www.un.org/disabilities. 
mentiert (DIM 2011). Deutschland hat sich, wie alle anderen Vertragsstaaten auch, dazu verpflichtet, Menschen mit Behinderungen einen gleichberechtigten Zugang zu einem inklusiven Bildungssystem zu gewährleisten (Aichele 2010), weshalb bei mangelnder Umsetzung der BRK in den Ländern eine Klärung vor dem Verfassungsgericht nötig wird (Aichele 2011).

Bei der Verwirklichung dieses Rechts haben die Vertragsstaaten sicherzustellen, dass Menschen nicht aufgrund einer Behinderung vom allgemeinen Bildungssystem und vom Besuch weiterführender Schulen ausgeschlossen werden. Menschen mit Behinderungen erhalten das Recht auf gleichberechtigten Zugang zu einem integrativen, hochwertigen und unentgeltlichen Unterricht an Grund- und weiterführenden Schulen mit angemessenen Vorkehrungen für ihre besonderen Bedürfnisse. Dies soll dadurch sichergestellt werden, dass Menschen die notwendige Unterstützung ihrer Bedürfnisse innerhalb des allgemeinen Bildungssystems erhalten.

Auch die Kultusministerkonferenz hat im Jahr 2010 in einer Stellungnahme Eckpunkte der bereitzustellenden barrierefreien inklusiven Bildungsangebote festgehalten: „Ein inklusiver Unterricht trägt der Vielfalt von unterschiedlichen Lern- und Leistungsvoraussetzungen der Kinder und Jugendlichen Rechnung. Es werden die Voraussetzungen dafür geschaffen, dass sie sich über eine Vielfalt an Handlungsmöglichkeiten selbstbestimmt und selbstgesteuert in ihren Entwicklungsprozess einbringen. Das Konzept des handlungsorientierten, ganzheitlichen Unterrichts soll den Kindern und Jugendlichen die notwendigen Erfahrungs- und Zugangsfelder für aktive, zunehmend selbstständige und ergebnisorientierte Entwicklungsprozesse bieten. Damit werden Grundlagen für ein nachhaltiges, lebenslanges Lernen gelegt [...] “ (KMK 2010b: 8-11).

\section{Fazit}

Die im vorliegenden Beitrag beschriebenen Kennzeichen der sonderpädagogischen Fördersysteme stehen in einem engen Zusammenhang mit dem Aufbau des deutschen Bildungswesens, seiner Gliedrigkeit und den Praktiken der Messung und Bewertung von Leistung sowie der Zuweisung und Überweisung an unterschiedliche Schultypen. Die Stratifizierung der Sekundarstufe bildet eine organisationelle Infrastruktur, die die Selektion von Schülern und Schülerinnen begünstigt, anstatt das Ziel des gemeinsamen Lernens zu stärken. Die bestehenden pädagogischen Institutionen und Disziplinen schaffen Diskurse um Begabung, Leistungsfähigkeit, Behinderung, und Förderbedarfe die der Legitimation sonderpädagogischer Fördersysteme dienen, die einem Großteil der förderbedürftigen Kinder und Jugendlichen keine Bildungserfolge garantiert. Der bildungspolitische Anspruch des deutschen Bildungswesens, die individuelle Förderung und gesellschaftliche Integration von Kindern und Jugendlichen durch schulische Segregation zu gewährleisten, kann als revidiert gelten. Die Bildungserfolge anderer demokratischer Staaten gehen u. a. auf das bildungspolitische Ziel einer Inklusion von 
allen Schülern und Schülerinnen an gemeinsamen Schulen zurück (Richardson/Powell 2011). Das Bildungswesen als ein Ort der Vielfalt verstanden, trägt zur gesellschaftlichen Integration aller bei, weil es die Aufmerksamkeit der Beteiligten auf mögliche kulturelle, soziale, psychische oder körperliche Gemeinsamkeiten und Unterschiede lenkt und diese in den Bildungsprozessen der Kinder und Jugendlichen produktiv macht (PreussLausitz 2001).

Der vorliegende Beitrag verdeutlicht, wie weit das deutsche Bildungswesen von der Forderung nach einer schulischen Inklusion aller Kinder und Jugendlichen entfernt ist. Das deutsche Bildungswesen wird der Forderung nach Inklusion in mehrfacher Hinsicht (noch) nicht gerecht: Im Vergleich zu den meisten europäischen Staaten schafft die Bildungspolitik in Deutschland bislang schulorganisatorische Voraussetzungen, die sich stark an dem Prinzip orientieren, Leistungshomogenität von Schülergruppen herstellen zu wollen. Im Gegensatz dazu orientieren sich viele andere europäische Staaten an den Prinzipien der Leistungsheterogenität und Individualisierung, indem sie einen großen Anteil von Schülern und Schülerinnen mit sonderpädagogischem Förderbedarf in den allgemeinen Schulen unterrichten und benachteiligte Kinder besonders unterstützen. Darüber sichern sie den Erfolg ihrer Bildungssysteme insgesamt. In Deutschland hingegen wurden im Jahr 2008 über 82 Prozent aller Schüler und Schülerinnen mit festgestelltem Förderbedarf in Sonderschulen unterrichtet. Ein ähnlich großer Anteil verließ die Schule ohne Abschluss.

Wie die Vergleiche der Bundesländer und der Nationalstaaten gezeigt hat, gibt es eine enorme Bandbreite sonderpädagogischer Fördersysteme. Förderung kann entweder nahezu ausschließlich in besonderen Einrichtungen angeboten werden oder - ganz im Gegenteil - an allen allgemeinbildenden Schulen. Es handelt sich somit nicht um eine Frage der Machbarkeit, die den Grad der schulischen Integration und die Einrichtung inklusiver Klassen und Schulen bestimmen, sondern um Fragen der bildungspolitischen Umsetzung international anerkannter Menschenrechte auf Bildung und gesellschaftliche Teilhabe.

\section{Literatur}

Aichele, Valentin (2010): Behinderung und Menschenrechte: Die UN-Konvention über die Rechte von Menschen mit Behinderungen. In: Aus Politik und Zeitgeschichte 23/2010, S. 13-19.

Aichele, Valentin (2011): Zur Rezeption der UN-Behindertenrechtskonvention in der gerichtlichen Praxis. In: AnwBl 10, S. 727-730.

Allmendinger, Jutta (1999): Bildungsarmut. Zur Verschränkung von Bildungs- und Sozialpolitik. In: Soziale Welt 5o, S. 35-50.

Baethge, Martin/Solga, Heike/Wieck, Markus (2007): Berufsbildung im Umbruch - Signale eines überfälligen Aufbruchs. Bonn/Berlin: Friedrich-Ebert-Stiftung. 
Begemann, Ernst (1970): Die Erziehung der sozio-kulturell benachteiligten Schüler. Hannover: Schroedel.

Boban, Ines/Hinz, Andreas (2003). Index für Inklusion. Lernen und Teilhabe in der Schule der Vielfalt entwickeln. Halle-Wittemberg: Martin-Luther-Universität.

Booth, Tony/Ainscow, Mel (2000): Index for Inclusion. Developing Learning and Participation in Schools. London: Centre for Studies on Inclusive Education (UK).

Cloerkes, Günther (2003): Zahlen zum Staunen. Die deutsche Schulstatistik. In: ders. (Hrsg.): Wie man behindert wird. Heidelberg: Winter, S. 11-25.

Degener, Theresia (2009): Die UN - Behindertenrechtskonvention als Inklusionsmotor. In: Recht der Jugend und des Bildungswesens 2/2009, S. 200-219.

DIM (2011): Stellungnahme der Monitoring-Stelle (31. März 2011), Eckpunkte zur Verwirklichung eines inklusiven Bildungssystems: Empfehlungen an die Länder, die Kultusministerkonferenz (KMK) und den Bund. Berlin: Deutsches Institut fur Menschenrechte.

EADSNE (2003). Special Education across Europe in 2003: Trends in Provision in 18 European Countries. Middelfart, DK: European Agency for Development in Special Needs Education.

Eberwein, Hans (2009): Zur Integrationsentwicklung in Deutschland in Schule und Kindergarten. In: Eberwein, H./Knauer, S. (Hrsg.), S. 504-513.

Eberwein, Hans/Knauer, Sabine (Hrsg.) (2009): Integrationspädagogik. Kinder mit und ohne Beeinträchtigung lernen gemeinsam. Weinheim: Beltz.

Ellger-Rüttgardt, Sieglind (2008): Geschichte der Sonderpädagogik. München: Reinhardt.

Eurydice (2005): Key Data on Education in Europe 2005, Brussels: Europäische Kommission.

Faulstich-Wieland, Hannelore (2004): Schule und Geschlecht. In: Helsper, W. (Hrsg.) Handbuch der Schulforschung. Wiesbaden: VS Verlag, S. 647-669.

Freitag, Walburga K. (2005): Contergan. Eine genealogische Studie des Zusammenhangs wissenschaftlicher Diskurse und biographischer Erfahrungen. Münster: Waxmann.

Füssel, Hans-Peter/Kretschmann, Rudolf (1993): Gemeinsamer Unterricht für behinderte und nicht-behinderte Kinder. Witterschlick: Wehle.

Füssel, Hans-Peter (2009): „Niemand darf wegen seiner Behinderung benachteiligt werden“. Die schulrechtlichen Rahmenbedingungen für eine integrative Pädagogik. In: Eberwein, H./ Knauer, S. 158-163.

Gabel, Susan/Curcic, Svjetlana/Powell, Justin J.W./Kader, Khaled/Albee, Lynn. Migration and Ethnic Group Disproportionality in Special Education. Disability \& Society 24(5): S. $625-639$.

Ginnold, Antje (2008): Der Übergang Schule - Beruf von Jugendlichen mit Lernbehinderung: Einstieg - Ausstieg - Warteschleife. Bad Heilbrunn: Klinkhardt.

Giroux, Henry (1997): Pedagogy and the Politics of Hope. Theory, Culture, and Schooling. Boulder, CO: Westview Press.

Goffman, Erving (1975): Stigma - Über Techniken der Bewältigung beschädigter Identität. Frankfurt am Main: Suhrkamp Verlag.

Hänsel, Dagmar (2005): Die Historiographie der Sonderschule. Eine kritische Analyse. In: Zeitschrift für Pädagogik 51(1), S. 101-114.

Hirschberg, Marianne (2009): Behinderung im internationalen Diskurs: Die flexible Klassifizierung der Weltgesundheitsorganisation. Frankfurt/Main: Campus.

Hofmann, Wolfgang (1930): Erhebungen über die Berufsfähigkeit entlassener Hilfsschüler. In: Die Hilfsschule 23, S. 132-146.

Hofsäss, Thomas (1993): Die Überweisung von Schülern auf die Hilfsschule und die Schule für Lernbehinderte in Deutschland seit 1918. Berlin: Marhold. 
KMK (2010a): Sonderpädagogische Förderung in Schulen 1999 bis 2008. Statistische Veröffentlichungen Dokumentation Nr. 189 - März 2010. Bonn: Ständige Konferenz der Kultusminister der Länder in der Bundesrepublik Deutschland.

KMK (2010b): „Inklusive Bildung von Kindern und Jugendlichen mit Behinderungen in Schulen“ (Entwurfsfassung, Stand: 03.12. 2010). Bonn: KMK.

Knauer, Sabine/Ramseger, Jörg (Hrsg.) (2009) Welchen Beitrag leistet die schulische Integration von Menschen mit Behinderungen auf dem Weg in den ersten Arbeitsmarkt? Berlin: Bundesministerium für Arbeit und Soziales.

Kornmann, Reimer (2003): Zur Überrepräsentation ausländischer Kinder und Jugendlicher in „Sonderschulen mit dem Schwerpunkt Lernen“. In: Auernheimer, G. (Hrsg.), Schieflagen im Bildungssystem. Opladen: Leske \& Budrich, S. 81-112.

Kottmann, Brigitte (2006): Selektion in die Sonderschule. Das Verfahren zur Feststellung von sonderpädagogischem Förderbedarf als Gegenstand empirischer Forschung. Bad Heilbrunn: Klinkhardt.

Krappmann, Lothar ([1971] 2005): Soziologische Dimensionen der Identität. Stuttgart: Klett.

Krappmann, Lothar/Leschinsky, Achim/Powell, Justin (2003): Kinder, die besonderer pädagogischer Förderung bedürfen. In: Cortina, K. S., et al. (Hrsg.): Das Bildungswesen in der Bundesrepublik Deutschland. Reinbek: Rowohlt, S. 755-786.

Marquardt, Regine (1975): Sonderschule - und was dann? Zur Situation von Sonderschülern auf dem Arbeitsmarkt und im Beruf. München: Aspekte Verlag.

Maschke, Michael (2008): Behindertenpolitik in der Europäischen Union. Lebenssituation behinderter Menschen und nationale Behindertenpolitik in 15 Mitgliedsstaaten. Wiesbaden: VS Verlag.

Mayer, Karl Ulrich (2009): Lebensverlauf. In: Solga, H./Powell, J./Berger, P. A. (Hrsg.), Soziale Ungleichheit. Klassische Texte zur Sozialstrukturanalyse. Frankfurt/Main: Campus, S. 411-426.

Möckel, Andreas (Hrsg.) (1998): Erfolg - Niedergang - Neuanfang. 100 Jahre Verband Deutscher Sonderpädagogik. München: Reinhardt.

Moser, Vera/Loeken, Hiltrud/Windisch, Matthias/Saalow, Michaela (2008): Sonderpädagogische Professionsforschung: Eine Skizze des Forschungsstands. In: Zeitschrift für Heilpädagogik 3/o8, S. 82-87.

Pfahl, Lisa (2004): Stigma-Management im Jobcoaching. Berufliche Orientierungen benachteiligter Jugendlicher (Diplomarbeit). Selbständige Nachwuchsgruppe Working Paper 1/2004. Berlin: Max-Planck-Institut für Bildungsforschung.

Pfahl, Lisa (2011): Techniken der Behinderung. Der deutsche Lernbehinderungsdiskurs, die Sonderschule und ihre Auswirkungen auf Bildungsbiografien. Bielefeld: transcript Verlag.

Pfahl, Lisa/Powell, Justin J.W. (2010): Draußen vor der Tür: Die Arbeitsmarktsituation von Menschen mit Behinderung. In: Aus Politik und Zeitgeschichte 23/2010: S. 32-38.

Pfahl, Lisa/Powell, Justin J. W. (2011): Legitimating School Segregation. The Special Education Profession and the Discourse of Learning Disability in Germany. Disability \& Society 26(4): S. 449-462.

Powell, Justin J. W. (2011): Barriers to Inclusion: Special Education in the United States and Germany. Boulder, CO: Paradigm Publishers.

Powell, Justin J. W./Pfahl, Lisa (2009): Ein kontinuierlicher deutscher Sonderweg? In: Knauer, S./ Ramseger, J. (Hrsg.), Welchen Beitrag leistet die schulische Integration von Menschen mit Behinderungen auf dem Weg in den ersten Arbeitsmarkt? Berlin: BMAS, S. 61-73.

Prengel, Annedore (2006): Pädagogik der Vielfalt. Wiesbaden: VS Verlag. 
Preuss-Lausitz, Ulf (2001): Gemeinsamer Unterricht Behinderter und Nichtbehinderter. In: Zeitschrift für Erziehungswissenschaft 4(2): S. 209-224.

Richardson, John G./Powell, Justin J. W. (2011): Comparing Special Education: Origins to Contemporary Paradoxes. Stanford, CA: Stanford University Press.

Seitz, Simone (2008): Leitlinien didaktischen Handelns. In: Zeitschrift für Heilpädagogik 59(6): S. 226-233.

Solga, Heike (2005): Ohne Abschluss in die Bildungsgesellschaft. Opladen: Verlag Barbara Budrich.

Stöppler, Thomas (2010): Ja zur Vielfalt (sonder-)pädagogischer Angebote. In: Aus Politik und Zeitgeschichte 23/2010: S. 19-24.

US DoED (2005): Annual Report to Congress on the Implementation of the Individuals with Disabilities Education Act. Washington, DC: U.S. Department of Education.

Wagner, Sandra J. (2005): Jugendliche ohne Berufsausbildung. Aachen: Shaker.

Werning, Rolf/Reiser, Helmut (2008): Sonderpädagogische Förderung. In: Cortina, K. S., et al. (Hrsg.), Das Bildungswesen in der Bundesrepublik Deutschland. Reinbek: Rowohlt, S. 505-539.

Wocken, Hans (2000): Leistung, Intelligenz und Soziallage von Schülern mit Lernbehinderungen. In: Zeitschrift für Heilpädagogik 12/2000, S. 492-503.

Wocken, Hans (2010): Über Widersacher der Inklusion und ihre Gegenreden. In: Aus Politik und Zeitgeschichte 13/2010: S. 25-31. 
Ullrich Bauer • Uwe H. Bittlingmayer

Albert Scherr (Hrsg.)

\section{Handbuch Bildungs- \\ und Erziehungssoziologie}

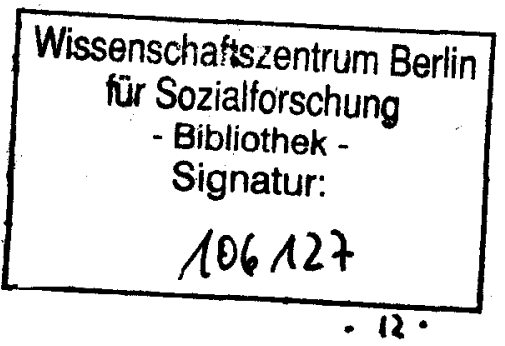


Der Verlag hat sich bemüht, alle uns bekannten Rechteinhaber zu ermitteln. Sollten dennoch Inhaber von Urheberrechten unberücksichtigt geblieben sein, bitten wir sie, sich mit dem Verlag in Verbindung zu setzen.

Die Deutsche Nationalbibliothek verzeichnet diese Publikation in der Deutschen Nationalbibliografie; detaillierte bibliografische Daten sind im Internet über http://dnb.d-nb.de abrufbar.

\section{Springer VS}

(C) VS Verlag für Sozialwissenschaften | Springer Fachmedien Wiesbaden 2012

Das Werk einschließlich aller seiner Teile ist urheberrechtlich geschützt. Jede Verwertung, die nicht ausdrücklich vom Urheberrechtsgesetz zugelassen ist, bedarf der vorherigen Zustimmung des Verlags. Das gilt insbesondere für Vervielfältigungen, Bearbeitungen, Übersetzungen, Mikroverfilmungen und die Einspeicherung und Verarbeitung in elektronischen Systemen.

Die Wiedergabe von Gebrauchsnamen, Handelsnamen, Warenbezeichnungen usw. in diesem Werk berechtigt auch ohne besondere Kennzeichnung nicht zu der Annahme, dass solche Namen im Sinne der Warenzeichen- und Markenschutz-Gesetzgebung als frei zu betrachten wären und daher von jedermann benutzt werden dürften.

Satz: text plus form, Dresden

Lektorat: Katrin Emmerich

Einbandentwurf: KünkelLopka GmbH, Heidelberg

Gedruckt auf säurefreiem und chlorfrei gebleichtem Papier

Springer VS ist eine Marke von Springer DE. Springer DE ist Teil der Fachverlagsgruppe Springer Science+Business Media. www.springer-vs.de 